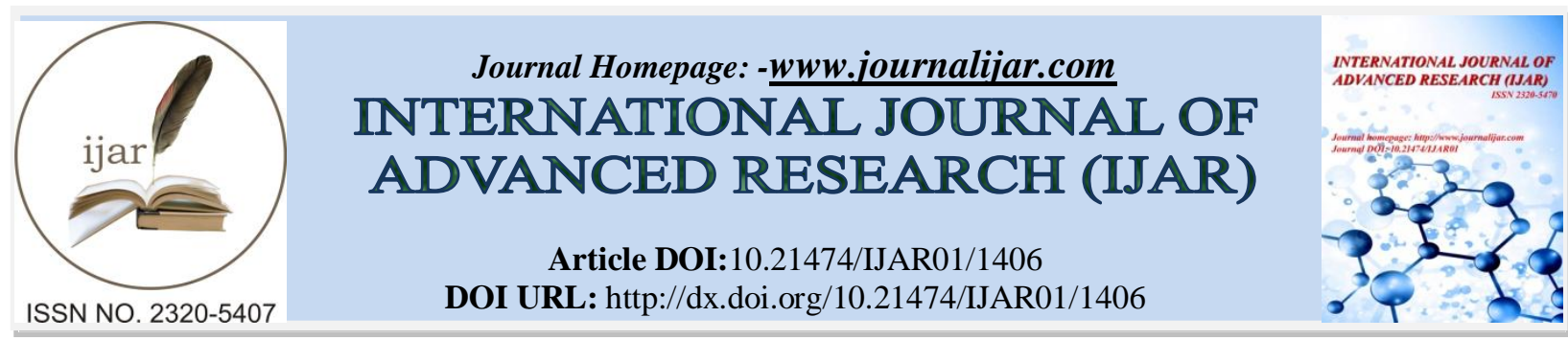

RESEARCH ARTICLE

\title{
ANTIBIOTIC SUSCEPTIBILITY PATTERN OF ASYMPTOMATIC Pseudomonas aeruginosa BACTERIURIA IN PREGNANT WOMEN ATTENDING A TERTIARY HEALTH CARE CENTRE IN MAKURDI, BENUE STATE CENTRAL NIGERIA.
}

*Aernan. P.T ${ }^{1}$, Umeh. E. $\mathbf{U}^{1}$ and Amali O. ${ }^{2}$.

1. Microbiology Unit, Department of Biological Sciences, Federal University of Agriculture, P M B 2373, Makurdi, Benue State, Nigeria.

2. Parasitology Unit of theDepartment of Biological Sciences, Federal University of Agriculture, P.M.B.2373, Makurdi, Benue State, Nigeria.

\section{Manuscript Info}

Manuscript History

Received: 18 June 2016

Final Accepted: 19 July 2016

Published: August 2016

Key words:-

Antibiotics, susceptibility,

Psedomonasaerugnosa, Makurdi, Central Nigeria.

\begin{abstract}
Epidemiological outcome studies have shown that infections caused by drug-resistant $P$. aeruginosa are associated with significant increases in morbidity. To determine resistance pattern of Pseudomonas aeruginosa isolated from asymptomatic bacteriuric women attending antenatal clinic in a tertiary hospital in Makurdi, Central Nigeria. Twenty eight(28) Pseudomonas aeruginosa isolates from a total of 277different bacterial organisms,were plated on Mueller Hilton agar following standard microbiological protocols. And the disc diffusion method of Kirby Bauer was adopted for susceptibility testing. Results showed that most of the isolates were not susceptible to the inhibitory activities of clindamycin (96.4\%), ceftriaxone (89.3\%) and clarithromycin $(89.3 \%)$ and susceptible at $100 \%$ to ofloxacin, perfloxacin and ciprofloxacin generally classified as the quinolones. Quinolones possess excellent activity invivo against enterobacteriaceae like $P$. aeruginosa amongst others.
\end{abstract}

Copy Right, IJAR, 2016,. All rights reserved.

\section{Introduction:-}

Pseudomonas aeruginosaurinary tract infections are usually hospital- acquired and are associated with catheterization, instrumentation, and surgery. These infections can involve the urinary tract through an ascending infection or through bacteraemia spread. In addition, these infections are a frequent source of bacteraemia. No specific characteristics distinguish this type of infection from other forms of urinary tract infection. Pseudomonas aeruginosa is the third most common pathogen associated with hospital-acquired catheter associated UTIs (Jarvis and Martone, 1992).

The genus Pseudomonas is made up of Gram-negative, rod-shaped bacteriathat inhabit many niches (Narins 2003; Laniniet al., 2011). Pseudomonas species are common inhabitants of the soil, water, and vegetation. The genus is particularly noteworthy because of the tendency of several species to cause infections in people who are already ill, or whose immune systems are not operating properly. Such infections are termed opportunistic infections (Narins 2003; Nikbinet al., 2012). Pseudomonas rarely causes infections in those whose immune systems are fully functional. The disease-causing members of the genus are therefore prevalent where illness abound. Pseudomonas

Corresponding Author:-Aernan. P.T. 
species are one of the major causes of nosocomial (hospital acquired) infections (Wolska and Szwada 2008; Narins 2003; Laniniet al., 2011). The species that comprise the genus Pseudomonas are part of the wider family of bacteria that are classified as Pseudomonadaceae. There are more than 140 species in the genus. The species that are associated with opportunistic infections include Pseudomonas aeruginosa, Pseudomonasmaltophilia, Pseudomonas fluorescens, Pseudomonas putida, Pseudomonas cepacia, Pseudomonas stutzeri, and Pseudomonas putrefaciens. Pseudomonas aeruginosa is probably the most well-known member of the genus (Narins 2003). The importance of Pseudomonas as a disease causing agent was not adequately recognised till recently, when it established itself as one of the most troublesome agents causing nosocomial infections (Shyamala and Rao 2015). Pseudomonas can grow on almost any available surface where enough moisture and nutrients are present (Wolska and Szwada 2009).

Pseudomonas aeruginosa presents a serious therapeutic challenge for treatment of both community-acquired and nosocomial infections, and selection of the appropriate antibiotic to initiate therapy is essential to optimizing the clinical outcome (Bontenet al., 1999). Unfortunately, selection of the most appropriate antibiotic is complicated by the ability of $P$. aeruginosa to develop resistance to multiple classes of antibacterial agents, even during the course of treating an infection. Epidemiological outcome studies have shown that infections caused by drug-resistant $P$. aeruginosa are associated with significant increases in morbidity, mortality, need for surgical intervention, length of hospital stay and chronic care, and overall cost of treating the infection. Even more problematic is the development of resistance during the course of therapy, a complication which has been shown to double the length of hospitalization and overall cost of patient care (Bisbeet al., 1988). P. aeruginosa can develop resistance to antibacterials either through the acquisition of resistance genes on mobile genetic elements (i.e., plasmids) or through mutational processes that alter the expression and/or function of chromosomally encoded mechanisms. Both strategies for developing drug resistance can severely limit the therapeutic options for treatment of serious infections.

Asymptomatic bacteriuria (ASB), or asymptomatic urinary infection (AUTI), is an isolation of a specified quantitative count of bacteria in an appropriately collected urine specimen obtained from a person without symptoms or signs referable to urinary infection (Nicolle et al., 2005). Urinary tract infections (UTIs) are characterized by the presence of infectious agents in the genito-urinary tract that cannot be explained by contamination. Infections of the kidney, ureter and bladder constitute UTI. When infections occur in the kidney and ureter it is called upper urinary tract infections (UUTI) and when it is from the bladder downwards it is called lower urinary tract infections, LUTI (Sundararajet al., 2004). These agents have the potential to invade the tissues of the urinary tract and adjacent structures (Bernard et al., 2011). UTI has been reported as the second most common infectious disease diagnosis, after pneumonia, leading to hospitalization, and it is the most common underlying cause of bacteremia (Ravehet al., 2006).

\section{Materials and Methods:- Sample Collection:-}

The study was carried out in a tertiary hospital in Makurdi, Middle belt region of Nigeria between September 2014 and April 2015. The hospital serves as a referral Centre for over half a million people within $40 \mathrm{~km}$ radius of the city. The town is divided by the River Benue into the north and south banks. Owing to its location in the Benue River, Makurdi experiences warm temperature most of the year.

A purposive selection consisting of pregnant women attending the ante-natal clinic was taken. This included women in the three trimesters of pregnancy. Patients were excluded if they had symptoms of urinary tract infection, had taken antibiotics during the previous week, or had any signs of labor.

A total of 438 pregnant women participated in this study.Written informed consent was obtained from the women before collection of each specimen, following standards from the ethical guidelines of the medical institution. Each of the women were instructed on how to collect a clean catch midstream urine sample in a sterile container. Following standard microbiological protocols for culture and Isolation of microorganisms, urine samples were streaked using sterile wireloop on nutrient agar, incubated at $37^{\circ} \mathrm{C}$ for $24 \mathrm{hrs}$, the cultural and morphologicalcharacteristics of distinct and isolatedcolonies were studied. This included size,elevation, opacity and colour. Thoseresembling Pseudomonas were inoculatedonto Cystine Lactose Electrolyte Deficiency (CLED) and coloniesthat did not ferment Lactose were presumptively identified as Pseudomonas aeruginosa andconfirmed by the oxidase slide and tubeagglutination tests. Pseudomonasaeruginosa are usually Oxidase positive. Plates were then viewed under UV rays to observe a characteristic fluorescent. Gram-negative rods were identified as lactose or non- 
lactosefermenters using Eosin Methylene Blue(EMBand MacConkey agar. Plates were further sub cultured on a selective medium using Pseudomonas Chromagar and Centimide agar, this was incubated for $24 \mathrm{hrs}$ at $37^{\circ} \mathrm{C}$. Plates were observed for green-mauve color on Pseudomonaschromagar and yellow green or yellow brown on centrimide agar, again plates were viewed under UV rays for fluorescence. Suspected organisms were inoculated on nutrient agar and incubated for $24 \mathrm{hrs}$ at $37^{\circ} \mathrm{C}$. Colonies from the nutrient agar were presented for the Gram stain, Antibiotic Sensitivity tests, Citrate, Indole, Urease, Oxidase and Rapid 32 API tests. Each isolate originating from a single colony of each patient's culture was identified as P. aeruginosa by analytical profile index test kit (API 20E test; bioMérieux.)

\section{Antimicrobial susceptibility test Discs:-}

A total of 28Pseudomonas aeruginosa strains from the different women across different geographical regions within Makurdi metropolis were employed in this study.The disc diffusion method of Bauer et al (1966) was used in this study. Five colonies of each strain of the isolate were suspended in a sterile bijou bottle containing $5 \mathrm{mls}$ of peptone water (Lab M) and incubated overnight at $37^{\circ} \mathrm{C}$. The overnight broth cultures were diluted to $10^{6}$ colony-forming units per ml. A sterile cotton-tipped applicator was introduced into standardized inoculum and used to inoculate dried plate of sensitivity test agar (STA) for each isolate. Antimicrobial susceptibility test discs were obtained from Oxoid Ltd, UK. They comprise:Ampicillin (PN) $10 \mu \mathrm{g}$, Ciprofloxacin (CPX) 10 $\mu \mathrm{g}$, Gentamicin (CN) $10 \mu \mathrm{g}$.

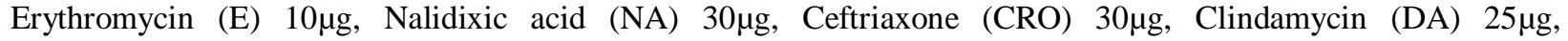

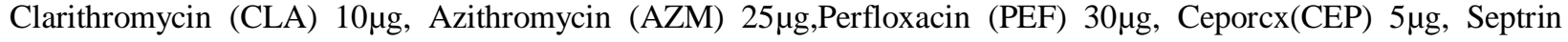

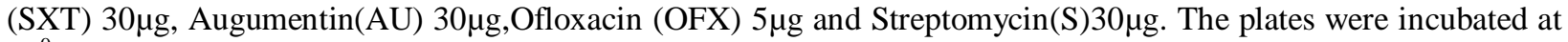
$37^{\circ} \mathrm{C}$ for $18-24$ hours. Zones of inhibition were measured and interpreted using the chart from Clinical Laboratory Standard Institute (CLSI), 2008.

\section{Results:-}

From 438 urine samples screened for ASB, 277 were positive for significant bacteriuria $\left(10^{5} \mathrm{cfu} / \mathrm{ml}\right)$ out of which a total of twenty-eight (28) were identified and confirmed as Pseudomonas aeruginosa isolates. Results showed that most of the isolates were not susceptible to the inhibitory activities of clindamycin (96.4\%), ceftriaxone (89.3\%) and clarithromycin (89.3\%) amongst others. They were generally $100 \%$ sensitive to ofloxacin, perfloxacin and ciprofloxacin generally classified as the quinolones by the clinical laboratory standard institute (CLSI, 2008) including gentamycin which was also $100 \%$ sensitive. See Table 1.

Table 1:- Antibiotics Sensitivity Pattern of Pseudomonas aeruginosa Isolates from urine.

\begin{tabular}{|l|l|l|l|}
\hline Antibiotics & Disc Content $(\mu \mathrm{g})$ & No. and \% Resistant & No. and \% sensitive \\
\hline Ofloxacin-OFX & 5 & $0(0.00 \%)$ & $28(100 \%)$ \\
\hline Perfloxacin-PEF & 30 & $0(0.00 \%)$ & $28(100 \%)$ \\
\hline Ciprofloxacin-CPX & 10 & $0(0.00 \%)$ & $28(100 \%)$ \\
\hline Augumentin-AU & 30 & $2(7.1 \%)$ & $26(92.9 \%)$ \\
\hline Gentamycin CN & 10 & $0(0.0 \%)$ & $28(100 \%)$ \\
\hline Streptomycin S & 30 & $0(0.0 \%)$ & $28(100.0 \%)$ \\
\hline Ceporcx-CEP & 5 & $1(3.6 \%)$ & $27(96.4 \%)$ \\
\hline Nalidilic Acid-NA & 30 & $3(10.7 \%)$ & $25(89.3 \%)$ \\
\hline Septrin-SXT & 30 & $2(7.1 \%)$ & $26(92.9 \%)$ \\
\hline Ampicillin-PN & 10 & $12(42.9 \%)$ & $16(57.1 \%)$ \\
\hline Erythromycin-E & 10 & $22(78.6 \%)$ & $6(21.4 \%)$ \\
\hline Clarithromycin-CLA & 10 & $25(89.3 \%)$ & $3(10.7 \%)$ \\
\hline Clindamycin-DA & 25 & $27(96.4 \%)$ & $1(3.6 \%)$ \\
\hline Azithromycin-AZM & 25 & $6(21.4 \%)$ & $22(78.6 \%)$ \\
\hline Ceftriaxone-CRO & 30 & $25(89.3 \%)$ & $3(10.7 \%)$ \\
\hline
\end{tabular}

Figure 1 describes the prevalence of Pseudomonas aeruginosa in relation to age distribution in pregnant women. Age group 31-40 years recorded the highest prevalence rate of 57.1\% while women in the less or equal to 20 years age group had the least prevalence rate of 3.6\%.Figure 2 shows the prevalence of Pseudomonas aeruginosa in relation to parity. Women carrying their first pregnancy (primi-gravida) recorded the highest prevalence of Pseudomonas aeruginosa with a rate of $35.7 \%$ while those carrying their second pregnancy had the least prevalence rate of $7.1 \%$. 
The women in their third trimester i.e. 7-9 months of pregnancy had the highest prevalence rate of 53.6\% while women in their first trimester had the lowest prevalence rate of $7.1 \%$ (Fig. 3). When the prevalence of Pseudomonas aeruginosa was associated with occupation as displayed on Figure 4 . The house-wives recorded the highest prevalence rate of $35.6 \%$ while the farmers had the least prevalence rate of $3.6 \%$.

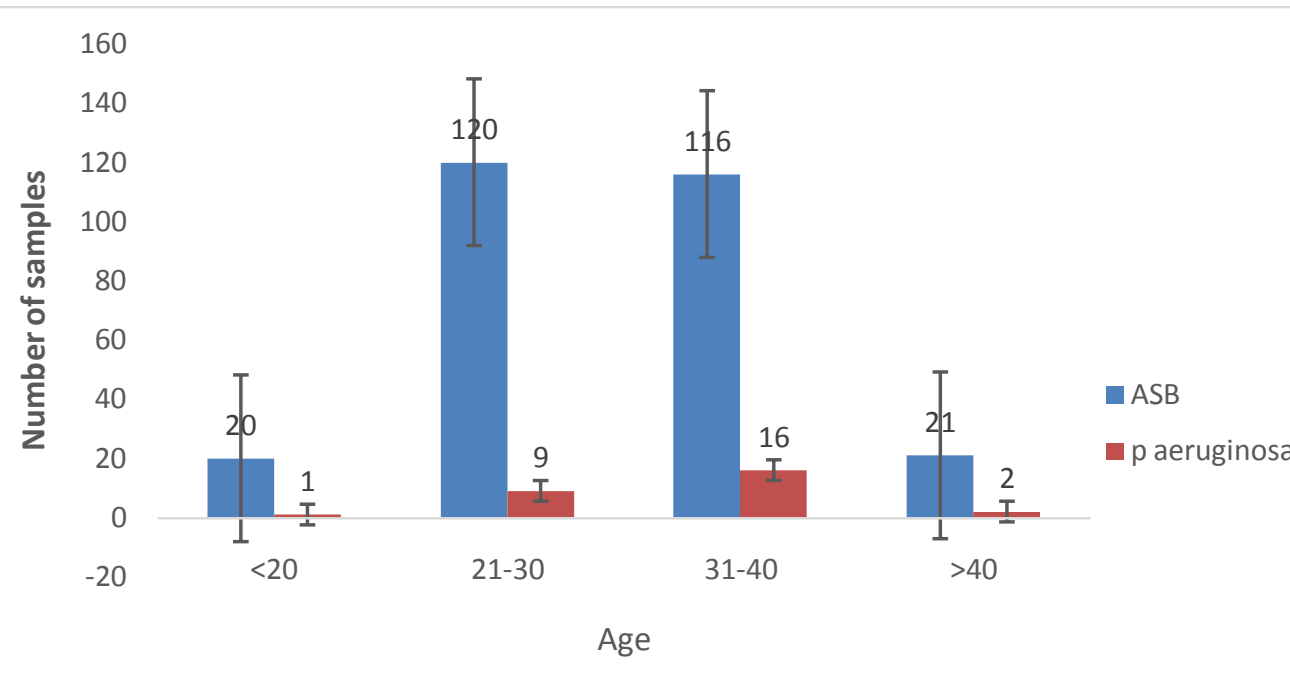

FIG 1: Prevalence of Psuedomonas aeruginosa in relation to age

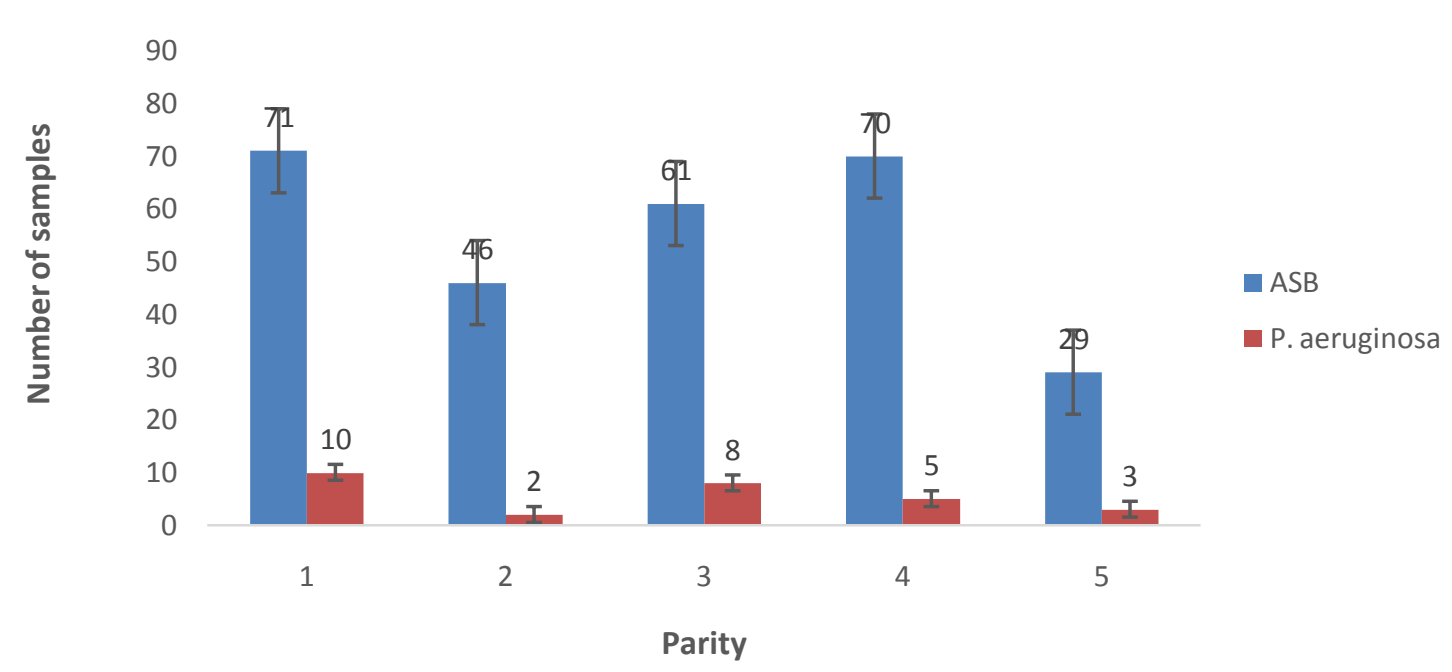

FIG 2: Prevalence of Pseudomonas aeruginosa in relation to parity (number of pregnancies) 

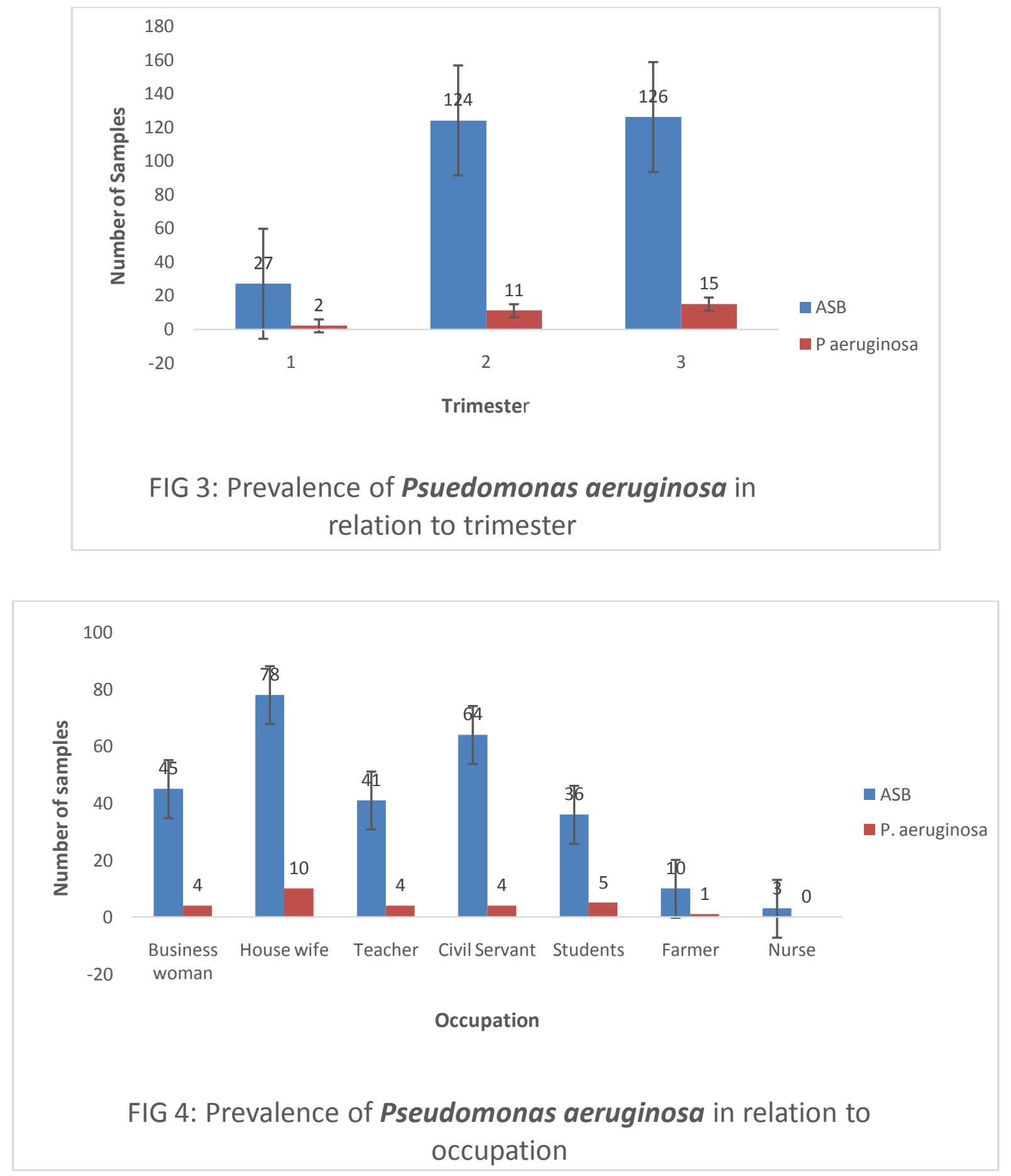

\section{Discussion:-}

The antibiotic sensitivity patterns from this study showed that all of the Pseudomonas aeruginosa isolated were sensitive to ofloxacin, perfloxacin, Gentamycin and streptomycin.Most of these very effective antibiotics,observed in this study belong to the quinolones class of antibiotics. Quinolones belong to a novel group of potent antibiotics biochemically related to nalidixic acid, which was developed initially as a urinary antiseptic but their use in pregnancy should be with caution (Delzell and Lefevre, 2000). Nalidixic acid, and its early analogs, oxolinic acid and cinoxacin, had limited clinical application as a result of widespread emergence of bacterial resistance. Newer quinolones have been synthesized by modifying the original two-ring quinolone nucleus with different side chain 
substitutions (Wolfson and Hooper, 1985). These new agents also known as fluoroquinolones, contain a fluorine atom attached to the nucleus. The choice of antibiotic should however be based on urine culture, stage of gestation, clinical data and the characteristics of the antibiotic. Although aggressive antibiotic treatment may be necessary to reduce the risk of pyelonephritis and other complications of asymptomatic bacteriuria in pregnancy, this should be done with caution as it known that urinary pathogens are becoming resistant to commonly used antibiotics which could be attributed to wide spread and indiscriminate use of the drugs (Okonko et al., 2009). A number of other fluoroquinolones including ofloxacin, enoxacin, lomefloxacin, temafloxacin, fleroxacin have now been released and used clinically. Quinolones possess excellent activity in vivo against enterobacteriaceae:- . aeruginosa, Citrobacter, Serratia, and Acinetobacter spp $\beta$-lactamase-postive and -negative $H$. Influenza, and Gram-negative cocci such as N.gonorrhoeae, N. Meningitidis, and Branhamelacatarrhalis (Wolfson and Hooper, 1985).

From this study P.aeruginosa exhibited a highlevel of resistance to erythromycin (78.6\%), clarithromycin (89.3\%), clindamycin (96.4\%) and ceftriaxone (89.3\%).

Erythromycin is a macrolide antibiotic derived from Streptomyces erythreus. Its chemical structure consists of a macrocyclic lactone ring attached to two sugar moieties, desosamine and cladinose. Other macrolides and those currently under investigation include dirthromycin, clarithromycin, roxithromycin, azithromycin and spiramycin (Hardy et al., 1988; Kirst and Sides, 1989). Erythromycin is a bacteriostatic agent that inhibits bacterial protein biosynthesis. This agent may be bactericidal at high drug concentration and against low inoculums of bacteria. It binds reversible to the 50S ribosomal subunits of susceptible microorganism, thereby blocking the translocation reaction of polypeptide chain elongation. Erythromycin is a relatively broadspectrum antibiotic with activity against Gram positive and some Gram negative bacteria, Mycoplasmas, Chlamydiae, Treponemas, and Rickettsiae (Washington and Wilson, 1985). Erythromyccin has however proved resistant in this present study. Pseudomonas can cause septic arthritis in pregnant subjects, the high occurrence of this organism in the middle aged women agrees with findings of Mittal and Wing, (2005). Women in the third trimester of pregnancy were observed to have the highest prevalence level of 15(53.6\%) than those in their second and first trimesters. This agrees with the findings of Lindsay (2003), who suggested that asymptomatic bacteriuria increases with increase in gestational period. In terms of occupational status, the housewives were observed to have a higher bacteriuria prevalence of $10(35.7 \%)$ than women across other occupation, which could be as a result of reduced social exposure and poor hygiene. The working class women are more educated, and enlightened perhaps taking more precautionary measures (which includes personal hygiene, etc) to avoid been infected. The age group 31-40 years had the highest prevalence of $16(57.1 \%)$, women in this age group are still sexually active and multiparity is also a risk factor in acquiring asymptomatic bacteriuria although Pseudomonas was associated more in women carrying their first pregnancy $10(35.7 \%)$.

\section{Conclusion:-}

In conclusion, $P$. aeruginosa were isolated from urine samples obtained from pregnant women for evaluation of asymptomatic bacteriuria in the obstetrics and gynecology unit of the health institution. Although these organisms, just like E. coli, indicate asymptomatic colonization, we identified certain risk factors for Pseudomonas bacteriuria. These data may allow for the adaptation of empiric protocols for the treatment of UTI in the obstetrics and gynecology department. In addition, it may assist clinicians in the selection of optimal antibiotic treatment for patients with these risk factors. This modified approach may contribute to improved patient care as well as more optimal use of antimicrobial agents.

\section{References:-}

1. Bauer AW, Kirby WWM, Sherris JC. Turk M.(1966) Antibiotic susceptibilitytesting by standardized single disc method. Am. J.Clin. Pathol. 45:493-496 Bisbe, J., J. M. Gatell, and J. Puig. (1988). Pseudomonas aeruginosa bacteremia: univariate and multivariate analyses of factors influencing the prognosis in 133 episodes. Rev. Infect. Dis. 10:629-635.

2. Bonten, M. J., D. C. Bergmans, H. Speijer, and E. E. Stobberingh. (1999).Characteristics of polyclonal endemicity of Pseudomonas aeruginosa colonization in intensive care units. Implications for infection control. Am. J. Crit. Care Med. 160:1212-1219.

3. Berard, A., Santos, F., Ferreira, E. and Perreault, S. (2011). Urinary Tract Infections during Pregnancy, Urinary Tract Infections, Dr. Peter Tenke (Ed.), ISBN: 978-953-307-757 4, InTech, Available from: http://www.intechopen.com/books/urinary-tract infections/urinarytract-infections-during-pregnancy 
4. Chen HY, Yuan M, Livermore DM. (1993). Mechanisms of resistance to Blactam antibioticsamongstPseudomonas aeruginosa isolates collected in the UK in 1993. J Med Microbiol 1995; 43:300-9.

5. Hardy, D.J, Hanson, C.W, Hensey, D.M, Beyer, J.M and Fernandes, P.B (1988): Susceptibility of Camphylobacter pylori to macrolides and fluoroquinolones. Journal of Antimicrobial therapy, 22(5): 631-636.

6. Jarvis, W.R and Martone, W.J. (1992): Predominant pathogens in hospitalinfections. Journal of Antimicrobial Chemotherapy 29: 19-24.

7. Kirst, H.A and Sides, G.D (1989): New directions for Macrolide antibiotics: Structural modifications and in vitro activity. Antimicrobial Agents Chemotherapy. 33: 1413-1418.

8. Lanini, S.,SilviaD’Arezzo, S., Puro, V., Martini1, L., Imperi, F., Piselli, P., Montanaro, M., S Paoletti, $\quad$ S., Visca, P. and Ippolito, G. (2011). Molecular Epidemiology of a Pseudomonas aeruginosa Hospital Outbreak Driven by a Contaminated Disinfectant Soap Dispenser. Plusone, 6(2): 1-10.

9. Mittal, P. and Wing, D.A. (2005) Urinary tract infections in pregnancy. Clinical Perinatology, 32, 749764.

10. Narins, B. (2003). World of Microbiology and Immunology. Volume 1 and 2. Gale Group $\quad$ Inc., $\quad$ Michigan, 465-466.

11. Nicolle LE, Bradley S, Colgan R, Rice JC, Schaeffer A, Hooton TM. (2005) Infectious Diseases Society of America guidelines for the diagnosis and treatment of asymptomatic bacteriuria in adults. Clin Infect Dis; 40:643-54.

12. Nikbin, V.S., Aslani, M.M., Sharafi, Z., Hashemipour, M., Shahcheraghi F. and Ebrahimipour, $\quad$ G.H. (2012). Molecular identification and detection of virulence genes among Pseudomonas aeruginosaisolated from different infectious origins. Iranian Journal of Microbiology, 4(3): 118-123.

13. Nordmann P, Guibert M.(1998). Extended-spectrum ß-lactamases in Pseudomonasaeruginosa. J AntimicrobChemother 1998; 42:28-31.

14. Nordmann P, Naas T. (1994). Sequence analysis of PER-1 extendedspectrum B-lactamasefrom Pseudomonas aeruginosa and comparison with class A B-lactamases. Antimicrob Agents Chemother 1994; 38:104-14.

15. Poirel L, Naas T, Guibert M, Chaibi EB, Labia R, Nordmann P.(1999). Molecular andbiochemical characterization of VEB-1, a novel class A extended-spectrum B-lactamase encoded by an Escherichia coli integron gene. Antimicrob Agents Chemother 1999; 43:573-81.

16. Naas T, Poirel L, Karim A, Nordmann P.(1999). Molecular characterization of In50, a class1 integron encoding the gene for the extended-spectrum B-lactamase VEB-1 in Pseudomonas aeruginosa. FEMS Microbiol Lett 1999; 176:411-9.

17. Raveh, D., Rosenzweig, I., Rudensky, B., Wiener-Well, Y. and Yinnon, A.M. (2006). Risk factors for bacteriuria due to Pseudomonas aeruginosa or Enterococcus spp inpatientshospitalized via the emergency department. European Journal of Clinical Microbiology and Infectious Disease, 25:331-334.

18. Shyamala, R. and Rao, J. (2015). Incidence of Pseudomonas species in urinary tract infection in a tertiary care hospital. Journal of Microbiology and Biotechnology Research, 5 (1):11-13.

19. Sundararaj, T., Anthoniraj, T., Kannan, N. and Muthukaruppan, S.M. (2004). Mirobiology for Higher Secondary. Tamil Nadu Text Book Corporation, India, $1^{\text {st }}$ Ed. Pp. 132.

20. Tribuddharat C, Fennewald M.(1999).Integron-mediated rifampin resistance inPseudomonas aeruginosa. Antimicrob Agents Chemother 1999; 43:960-2Vahaboglu H, Ozturk R, Aygun G, Coskunkan F, Yaman A, Kaygusuz A, et al.(1997):Widespread detection of PER-type extendedspectrum ß-lactamases among nosocomial Acinetobacter and Pseudomonas aeruginosa isolates in Turkey: a nationwide multicenter study. Antimicrob Agents Chemother 1997; 41:2265-9.

21. Washington, J.A.H and Wilson, W.R (1985): Erythromycin: a microbial andclinical perspective after 30 years of clinical use. Mayo Clinic Proceedings, 60: 189203, 271-278.

22. Wolska, K. and Szweda P.(2008). A comparative evaluation of PCR Ribotyping and ERICPCRfor determining the diversity of clinical Pseudomonas aeruginosa isolates. Pol J. Microbiol. 27: 320-326 Wolska, K. and Szweda, P. (2009). Genetic features of clinical Pseudomonas aeruginosa strains. Polish Journal of Microbiology, 58(3): 255-260.

23. Wolfson, J.S, and Hooper, D.C (1985): The fluroquinoles: structures, mechanisms of action and resistance, and spectra of activity in vitro. Antimicrobial Agents Chemotherapy, 28: 581-586. 